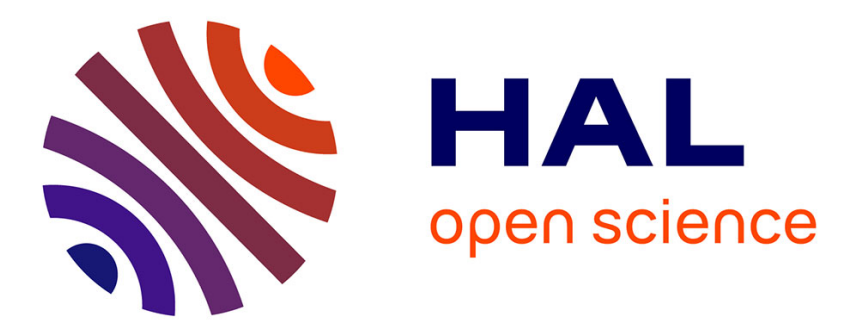

\title{
Compton Large Area Silicon Timing Tracker for Cosmic Vision M3
}

F. Lebrun, F. Aharonian, V. Beckmann, W. Bertoli, J.-M. Brom, A. Bykov, S. Brandt, T. Bulik, C. Budtz-Jorgensen, I. Caballero, et al.

\section{- To cite this version:}

F. Lebrun, F. Aharonian, V. Beckmann, W. Bertoli, J.-M. Brom, et al.. Compton Large Area Silicon Timing Tracker for Cosmic Vision M3. 8th INTEGRAL Workshop "The Restless Gamma-ray Universe", Sep 2010, Dublin, Ireland. pp.34. in2p3-00671014

\section{HAL Id: in2p3-00671014 https://hal.in2p3.fr/in2p3-00671014}

Submitted on 16 Feb 2012

HAL is a multi-disciplinary open access archive for the deposit and dissemination of scientific research documents, whether they are published or not. The documents may come from teaching and research institutions in France or abroad, or from public or private research centers.
L'archive ouverte pluridisciplinaire HAL, est destinée au dépôt et à la diffusion de documents scientifiques de niveau recherche, publiés ou non, émanant des établissements d'enseignement et de recherche français ou étrangers, des laboratoires publics ou privés. 


\section{CAPSiTT: a sensitive $100 \mathrm{keV}-100 \mathrm{MeV}$ all sky survey}

F. Lebrun ${ }^{1}$, F. Aharonian ${ }^{2,9}$, V. Beckmann ${ }^{1}$, W. Bertoli ${ }^{1}$, J.-M. Brom ${ }^{3}$, A. Bykov ${ }^{4}$, S. Brandt $^{5}$, T. Bulik ${ }^{6}$, C. Budtz-Jorgensen ${ }^{5}$, I. Caballero ${ }^{7}$, J. Cohen-Tanugi ${ }^{11}$ A. Creusot $^{1}$, F. Daigne ${ }^{8}$, M. Denis ${ }^{9}$, L. Drury ${ }^{10}$, Y. Gallant ${ }^{11}$, N. Gehrels ${ }^{12}$, U. Goerlach ${ }^{3}$, D. Götz ${ }^{7}$, C. Hamadache ${ }^{13}$, W. Hermsen ${ }^{14}$, J. Kiener ${ }^{13}$, L. Kuiper ${ }^{14}$, G. Lamanna ${ }^{15}$, P. Laurent $^{1}$, O. Limousin ${ }^{7}$, F. Mattana1, A. Marcowith ${ }^{11}$, T. Neubert ${ }^{16}$, C. Olivetto ${ }^{1}$, P. Orleanski $^{9}$, E. Parizot ${ }^{1}$, J.-L. Pinçon ${ }^{17}$, T. Pradier ${ }^{3}$, S. Schanne ${ }^{7}$, N. de Séréville ${ }^{18}$, S. Soldi ${ }^{7}$, M. Renaud ${ }^{11}$, M. Tagger ${ }^{17}$, V. Tatischeff ${ }^{13}$, R. Terrier $^{1}$, R. Walter ${ }^{19}$, S. Watanabe $^{20}$, J. Zurita Heras ${ }^{1}$

${ }^{I}$ APC/IN2P3-IRFU,10, rue A. Domon et L. Duquet, 75205, Paris, France

${ }^{2}$ MPI, Walther-Bothe-Laboratorium, P.O. Box 103980, D-69029 Heidelberg, Germany

${ }^{3}$ IPHC, 23, rue du loess - BP28 - 67037, Strasbourg Cedex 2, France

${ }^{4}$ Ioffe Physico-Technical Institute, 26 Polytekhnicheskaya, St Petersburg 194021, Russia

${ }^{5}$ DTU Space, Juliane Maries Vej 30DK - 2100 Copenhagen, Denmark

${ }^{6}$ Astronomical Observatory, The University of Warsaw, Al. Ujazdowskie 4, 00-478 Warsaw, Poland

${ }^{7}$ AIM/IRFU,CEA/Saclay, 91191 Gif sur Yvette, Saclay, France

${ }^{8}$ IAP, 98bis, bd Arago - F-75014 Paris, France

${ }^{9}$ CBK, Bartycka 18A, Warsaw, Poland

${ }^{10}$ DIAS, 10 Burlington Road, Dublin, Ireland

${ }^{11}$ LPTA, 13 place Eugène Bataillon 34095, Montpellier, France

${ }^{12}$ GSFC, 8800 Greenbelt Rd, Greenbelt, MD 20706, U.S.

${ }^{13}$ CSNSM, Bâtiment 104, 91405 Orsay Campus, France

${ }^{14}$ SRON, Sorbonnelaan 2, Utrecht, Netherland

${ }^{15}$ LAPP, 9 Chemin de Bellevue - BP 110, 74941, Annecy le Vieux CEDEX, France

${ }^{16}$ DMI, Lyngbyvej 100, DK-2100 Copenhagen, Denmark

${ }^{17}$ LPC2E, 3A Av. de la Recherche Scientifique, 45071 ORLEANS CEDEX 2, France

${ }^{18}$ IPNO, 15 Rue Georges Clemenceau, 91400 Orsay, France

${ }^{19}$ ISDC, Chemin d'Écogia 16, CH-1290, Versoix, Switzerland

${ }^{20}$ ISAS/JAXA, 3-1-1 Yoshinodai, Sagamihara, Kanagawa 229-8510, Japan

Proposed in response to the ESA call for the third Medium size mission (M3), CAPSiTT is a small mission designed for a 3-year survey of the non-thermal high energy sky from an equatorial LEO orbit. With a large effective area and a very wide field of view, its single instrument, a silicon tracker, provides good imaging, spectroscopic and polarimetric capabilities with a sensitivity 10-100 times better than COMPTEL. Nucleosynthesis and particle acceleration mechanisms in various sites are the main scientific topics addressed by CAPSiTT.

8th INTEGRAL Workshop

"The Restless Gamma-ray Universe"

Dublin, Ireland, September 27-30, 2010

\footnotetext{
${ }^{*}$ Speaker
} 


\section{Introduction}

The answers to many key questions regarding the most violent cosmic phenomena and their implications on the general evolution of the Universe are to be found in the $0.1-100 \mathrm{MeV}$ photon energy domain. First of all, massive stars and supernovae are the furnaces where most elements are formed and the nuclear lines emitted by radioisotopes synthesized in these objects are fundamental observables to understand stellar nucleosynthesis and the physics of supernova explosion. The most energetic of these stellar explosions, gamma-ray bursts, are visible up to very high redshifts and some are likely to mark the death of the first stars in the Universe. Gamma-ray bursts emit most of their energy in the $0.1-1 \mathrm{MeV}$ range, which contains the key to the relativistic ejection phenomena taking place during the explosion. Similarly, the most luminous jets powered by supermassive black holes in the Universe also radiate their energy in the $\mathrm{MeV}$ domain. Gamma-ray astronomy in this domain provides a unique way to look at the most distant and massive black holes and understand how they were formed in the early Universe. Gamma-ray astronomy below $100 \mathrm{MeV}$ is also the best tool to study low energy cosmic rays in the Galaxy, given that these particles cannot be detected near Earth because of the solar modulation. Finally, the physics of the extreme environments of collapsed objects have unique signatures in the $\mathrm{MeV}$ regime, in particular through polarization of their radiation. A polarimetric all-sky survey in the $0.5-100 \mathrm{MeV}$ range, with a sensitivity improvement of more than one order of magnitude over previous missions, is therefore fundamental for the study of the evolving violent Universe. But it also proves to be invaluable in the study of matter in extreme states and the exploration of the limits of contemporaneous physics.

In the last decade, the X-ray domain, the high and very high energy gamma-ray domains have seen the advent of very sensitive instruments such as XMM-Newton, Chandra, Fermi/LAT, HESS, MAGIC and Veritas. However, in the $0.1-100 \mathrm{MeV}$ energy range the progress has been much more limited; in fact no experiment achieved a survey sensitivity better than the COMPTEL instrument of the CGRO mission $(1991-2000)$. In the future, IXO and CTA will even increase the contrast. This situation is certainly not the result of a lack of activity on the experimental side, but is due to the difficulties inherent to this energy domain, where the probability of photon interaction with matter reaches a minimum while the instrumental background induced by cosmic-ray particles is very high.

It is generally agreed that the best approach for achieving a real sensitivity leap over a broad energy range in the $\mathrm{MeV}$ domain is to use the Compton imaging technique pioneered by COMPTEL. Major improvements over this instrument are now possible thanks to the development of advanced position-sensitive detectors for high-energy physics. Thus, whereas COMPTEL employed two separate detectors, a scatterer designed to favour Compton interactions and a calorimeter to absorb the scattered photons, the development of double-sided Si-strip tracking detectors now allows us to propose a Compton telescope without a calorimeter, i.e. a much lighter instrument based on a single technology. Furthermore, the fine spectral and position resolutions of modern Si detectors enable a gain in detection efficiency by about two orders of magnitude over COMPTEL, together with a very efficient background rejection. Other advantages of the proposed Si tracker include its high capability in measuring polarization - a 
key observable at high energies in the future - and its very large field of view. Last but not least it will also work well as a pair telescope and thus will have an energy coverage overlapping that of the Fermi/LAT. This Compton And Pair Silicon Timing Tracker (CAPSiTT) will be less efficient than the LAT at high energies, but, without tungsten converter plates, will have good polarimetric capabilities and a better angular resolution.

To achieve the best sensitivity, CAPSiTT should be launched into a circular Low Earth Orbit (LEO) with less than $5^{\circ}$ inclination to avoid the South Atlantic Anomaly, and operate in an automatic all-sky scanning mode, as exemplified by the recent success of Fermi/LAT at higher energies. Such a scanning strategy is also vital to deal with the extremely transient nature of the sky at these wavelengths. It is all in all possible to open a new window into the evolving violent Universe with a mission as simple as possible and significantly smaller than standard medium-class missions.

\section{The instrument}

CAPSiTT is a combined Compton and pair telescope taking full advantage of recent progresses in silicon detectors and microelectronics. Its main element is a large tracker $(1.2 \times 1.2 \times 0.9 \mathrm{~m})$ made of a stack of double-sided $2 \mathrm{~mm}$ thick $10 \times 10 \mathrm{~cm}$ silicon stripped detectors (DSSDs). There are 64 strips with a pitch of $1.5 \mathrm{~mm}$ on each side. To reduce the number of channels, the DSSDs are chained $6 \times 6$ on both directions, forming square Detection Units of 36 DSSDs readout by 12 low-noise, low-power (1 $\mathrm{mW} / \mathrm{channel})$ 64channels ASICs. The detection units are glued together by 5 to form stiff Modules equipped with an encoder and an FPGA controlling the 60 ASICs (see Fig. 2-1). The detection units are spaced vertically by $1 \mathrm{~cm}$. There are 4 towers of 16 modules each (see Fig 2-2) providing a stopping power is equivalent to $16 \mathrm{~cm}$ of silicon. To minimize the passive material inside the tracker, readout ASICs are located at the outskirts of the tracker and directly connected to the heat pipes that will convey the $250 \mathrm{~W}$ to a large radiator covering one side of the platform. A Module FPGA controls 60 ASICs and transfers their data to the Central Unit where

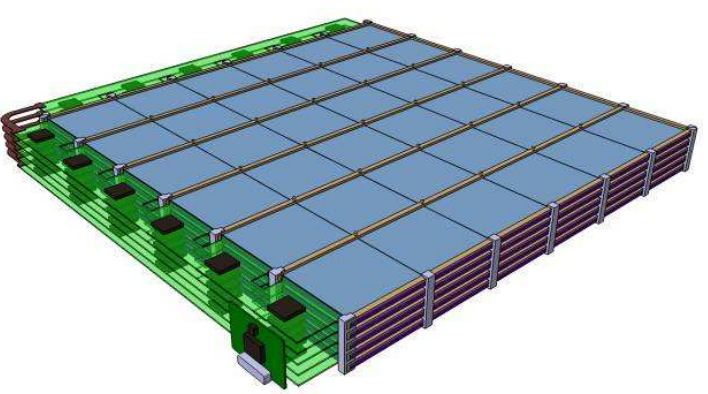

Figure 2-1: CAPSiTT Module

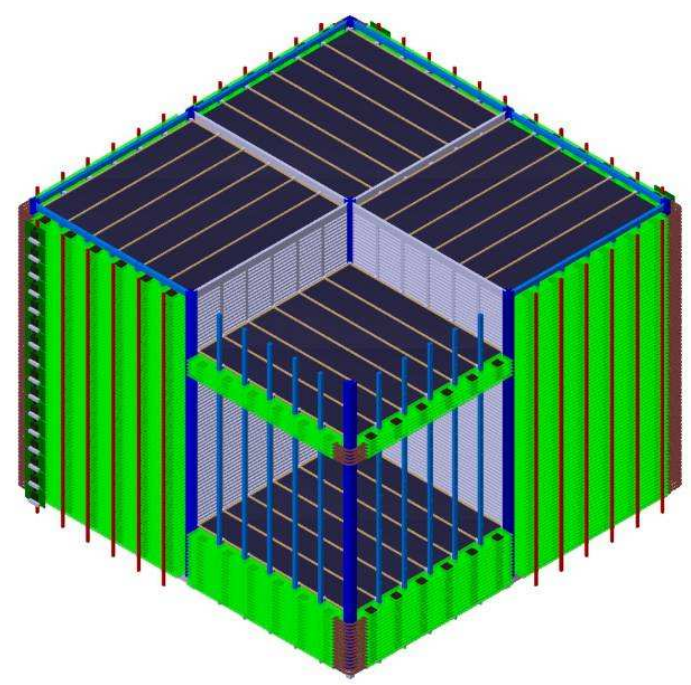

Figure 2-2: CAPSiTT tracker coincidences are performed. The tracker is complemented by a plastic anti-coincidence system similar to the one of the Fermi/LAT to reject the charged cosmic-ray background. CAPSiTT 
employs the Compton imaging technique pioneered by the COMPTEL instrument of the CGRO mission. But in contrast to this first-generation instrument, the fine position and spectral resolutions of the DSSDs now allow the design of a Compton telescope working well without a heavy calorimeter. Below $500 \mathrm{keV}$ it uses two interactions to retrieve the energy and the direction of the incoming photons while above this energy, the use of 3 interactions removes the need of a complete photon absorption [5] difficult to achieve without a calorimeter. Above 10 $\mathrm{MeV}$ CAPSiTT measures the paths of the electrons and positrons produced by pair conversion of incident $\gamma$-rays, like in the LAT. But in CAPSiTT the detected pair creations take place in the silicon-strip detectors themselves; there is no high- $\mathrm{Z}$ material converter.

\section{Performance and science goals}

With its survey strategy, CAPSiTT has been designed to open the window on the $100 \mathrm{keV}$ $100 \mathrm{MeV}$ sky. Its gain in sensitivity with regard to SPI and COMPTEL (see Table 3-1 and Fig. 3-1), particularly near $1 \mathrm{MeV}$, promises a huge discovery potential, a step forward that can be compared with that accomplished at higher energies by Fermi/LAT with regard to EGRET.

CAPSiTT will have the required sensitivity to provide a good census of present and past supernova explosions through the emission of various associated radioactivity lines $\left({ }^{56} \mathrm{Co},{ }^{44} \mathrm{Ti}\right.$, ${ }^{26} \mathrm{Al},{ }^{60} \mathrm{Fe}$ ), to study from this emission how stars explode and how elements are synthesized in cosmic explosions, and therefore to understand in detail the history of supernovae in our Galaxy and in the local group of galaxies. Despite its relative simplicity, CAPSiTT's performances are well within the requirements of the main science objectives in high-energy astrophysics. The energy resolution is more than enough to address most of the issues related to gamma-ray line astronomy. Even in the case of narrow lines (511 $\mathrm{keV}, 1.8 \mathrm{MeV}$ ), the gain in sensitivity is such that the line barycentre will be determined with the same accuracy as the INTEGRAL Spectrometer (SPI). In most astrophysical sites ( $\mathrm{SNe}$, novae, SNRs, hot plasmas) the lines are broad and the ultimate spectral resolution of SPI is not an advantage. For example, with a sensitivity improvement by a factor of about 70 over SPI for the detection of the $847 \mathrm{keV}{ }^{56} \mathrm{Co}$ line

\begin{tabular}{|l|r|r|r|}
\hline $\mathbf{E}(\mathbf{M e V})$ & $\boldsymbol{\Delta E}(\mathbf{k e V})$ & $\begin{array}{c}\text { CAPSiTT } \\
\left(\mathbf{c m}^{-2} \mathbf{s}^{-1}\right)\end{array}$ & $\begin{array}{c}\text { SPI } \\
\left(\mathbf{c m}^{-2} \mathbf{s}^{-1}\right)\end{array}$ \\
\hline 0.511 & 1.3 & $510^{-6}$ & $4.910^{-5}$ \\
\hline 0.847 & 35 & $3.110^{-6}$ & $2.210^{-4}$ \\
\hline 1.157 & 15 & $2.110^{-6}$ & $9.110^{-5}$ \\
\hline 1.809 & 2.0 & $1.610^{-6}$ & $1.010^{-4}$ \\
\hline 2.223 & 20 & $1.710^{-6}$ & $1.010^{-4}$ \\
\hline 4.438 & 100 & $2.510^{-6}$ & $1.110^{-4}$ \\
\hline
\end{tabular}

Table 3-1: CAPSiTT $3 \sigma$ line sensitivity $\left(10^{6} \mathrm{~s}\right)$

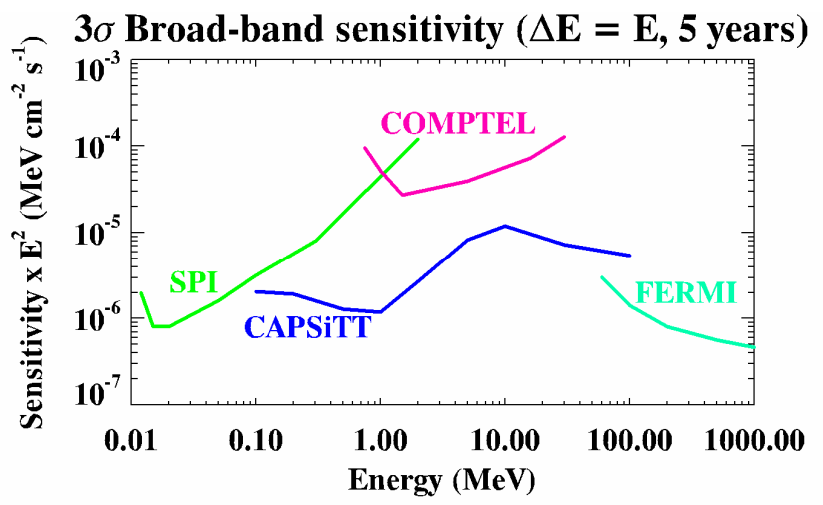

Figure 3-1 CAPSiTT survey sensitivity compared with past and on-going missions 
produced by thermonuclear supernovae, CAPSiTT will be able to detect at least 15 type Ia supernovae of all subtypes during its three years nominal mission time and measure for the first time the amount of ${ }^{56} \mathrm{Ni}$ synthesized in these objects. With its sensitivity and an energy resolution of $1 \%$ at the ${ }^{44} \mathrm{Ti}$ line energy of $1.157 \mathrm{MeV}$, CAPSiTT will be able to study the ejecta velocity distribution in a dozen of young galactic supernova remnants yet to be discovered. With an energy range covering three orders of magnitude, from $100 \mathrm{keV}$ to $100 \mathrm{MeV}$, CAPSiTT will be able to identify the high-energy emission mechanisms at work in supernova remnants and shed new light on the non-thermal hadronic content of these objects. With a very large field of view resulting in more than $40 \%$ of the MeV sky being covered at any moment, CAPSiTT will detect up to 15000 blazars, some of them at cosmological distances and will allow detailed studies of the evolution of supermassive black holes in the Universe and explore the history of jet emission in active galactic nuclei to more than 12 billion years before our time.

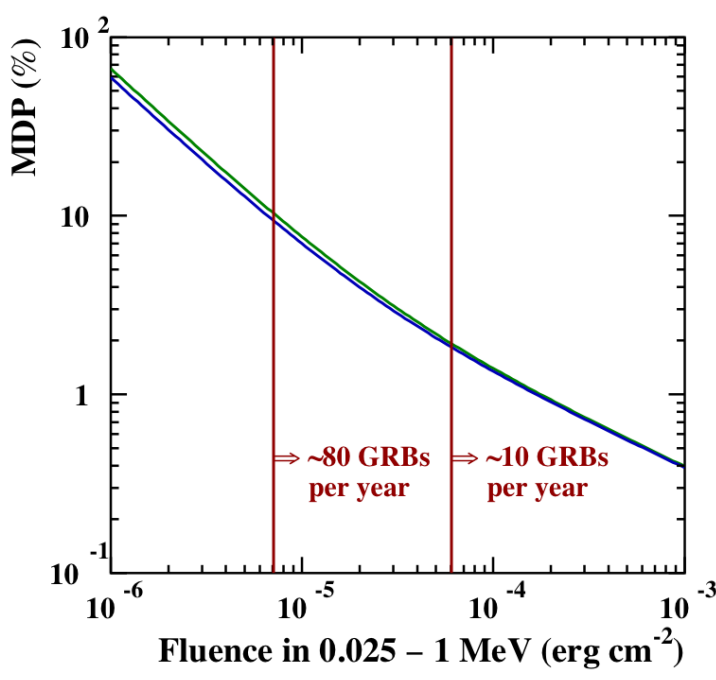

Figure 3-2: Minimum polarization detectable by CAPSiTT in the range $0.2-2 \mathrm{MeV}$ as a function of the GRB fluence. The assumed $G R B$ duration is $50 s$ and the source spectrum is a Band law with $\alpha=-1.1, \beta=-2.3$ and $E_{\text {peak }}$ $=200 \mathrm{keV}$ (green curve) or $1 \mathrm{MeV}$ (blue curve).
Last but not least, thanks to its high polarimetric capability and its large effective area, CAPSiTT will provide new strong observational constraints on the origin of the $\gamma$ ray emission from a variety of high-energy sources. For example, it will be able to measure a polarization degree higher than $10 \%$ in about 240 gamma-ray bursts in three years (see Fig. 3-2), thus shedding new light on the nature, geometry and magnetization of the ultra-relativistic outflow which produces this extreme emission. It will also constrain physical processes at work in the ejection phenomenon close to accreting black-holes, and determine the sites of $\gamma$-ray production in pulsar magnetospheres.

CAPSiTT will have the capability to detect for the first time nuclear lines emitted in the vicinity of neutron stars [1] and to provide stringent constraints on the dense matter equation of state. It will also study the high-energy phenomena associated with the extreme magnetic fields of a number of neutron stars.

The CAPSiTT performances will allow the detection of the $2.2 \mathrm{MeV}$ line from the inner accretion disc around black holes due to the neutrons capture by the fast protons.

CAPSiTT will be able to detect for the first time nuclear lines induced by low energy cosmic-rays in the interstellar medium [2] and will thus provide the first unambiguous measurement of this component in the Galaxy.

CAPSiTT will observe the Sun and the Earth as well and will be able to study particle acceleration during solar flares and terrestrial $\gamma$-ray flashes [3]. 
CAPSiTT will also put fundamental theories to a test. In particular, polarimetric measurements in the $\mathrm{MeV}$ range will be the most stringent tests on a possible Lorentz invariance violation that is expected by quantum gravity theories [4].

At the time of its launch, CAPSiTT will take its full role among a number of major observatories. It will look for the electromagnetic counterparts of the LISA detected merger events. It will provide high-energy spectral and polarimetric measurements to SVOM detected GRBs. It will provide an all-sky monitor for small field of view instruments such as the Cerenkov Telescope Array (CTA) as well as vital spectral information for broad band gammaray studies. It will also be a useful complement to forthcoming neutrino telescopes, in particular regarding supernova physics.

\section{The mission profile}

Being much lighter than the previously flown or envisioned Compton telescopes, CAPSiTT, with an estimated maximal payload mass of $970 \mathrm{~kg}$, can be launched from Kourou by a VEGA rocket into an equatorial (inclination $<5^{\circ}$ ) low Earth orbit (550 km altitude), which offers the best space environmental conditions for a $\gamma$-ray experiment. The satellite will be always pointed at the zenith (in the terrestrial equator plane) within a few degrees. Given the large field of view of the instrument, this most simple scanning strategy will achieve full sky coverage at $1 \mathrm{MeV}$ at each revolution (i.e. every 90 minutes). The mission operations, that will take place from ESA's European Space Operation Centre, will be restricted to the satellite control, the instrument TCs uploads and the telemetry downloading every 3 orbits (every 4.5 hours). The content of the onboard memory will be downloaded to Kourou station at a rate of $300 \mathrm{Mbit} / \mathrm{s}$. The total power budget of the CAPSiTT mission will amount to $1.1 \mathrm{~kW}$ and will be provided by one $10 \mathrm{~m}^{2}$ GaAs Solar Array Wing equipped with a Solar Array Drive Mechanism. We propose to use for the CAPSiTT spacecraft the SENTINEL-3 platform.

CAPSiTT will not be an observatory-type mission, but will always operate in a scanning mode. The Science Data Centre will be in charge of the instrument health monitoring, the quick look analysis and the data processing, archiving and distribution. After a year period allocated to the CAPSiTT Co-I team to fix the analysis software and exploit the first results, all data will become public as soon as possible.

\section{References}

[1] Çalişkan, Ş., et al. 2009, ApJ, 694, 593.

[2] Indriolo, N., et al. 2009, ApJ, 694, 257.

[3] Marisaldi, M. et al. 2010, Phys. Rev. Letters, 105, Issue 12

[4] Maccione, L., et al. 2008, Phys. Rev. D, 78, 103003

[5] Kroeger, R.A. et al., 2003, SPIE 4851, 1236 